\title{
Síntesis de nopol con Sn-SBA-15 y Sn-MCM-41
}

\section{Nopol synthesis over Sn-SBA-15 and Sn-MCM-41}

\author{
Luis F. Correa*, Edwin A. Alarcón*, Aída L. Villa ${ }^{*}$ \\ "Facultad de Ingeniería, Departamento de Ingeniería Química Universidad de Antioquia, Grupo \\ Catálisis Ambiental, Centro de Excelencia CENIVAM, Medellín, Colombia \\ §alvilla@udea.edu.co,ealarcon@udea.edu.co,fc.luis@gmail.com
}

(Recibido: Diciembre 19 de 2011 -Aceptado: Mayo 15 de 2012 -Versión Final: Junio 25 de 2012)

\section{Resumen}

Se comparó la actividad de Sn-MCM-41 y Sn-SBA-15 para la síntesis de nopol a partir de $\beta$-pineno y paraformaldehído. Los materiales se prepararon por los métodos hidrotérmico y el de impregnación húmeda incipiente, utilizando cloruro estañoso como sal precursora de Sn. Los catalizadores sintetizados por impregnación presentaron mayor actividad y fueron más selectivos a nopol que los materiales obtenidos por el método hidrotérmico. La conversión de $\beta$-pineno obtenida con Sn-MCM-41 fue similar a la obtenida con Sn-SBA-15. Mediante síntesis hidrotérmica, se obtuvo especies de Sn(IV) en forma de agregados de $\mathrm{SnO}_{2}$ y substituyendo isomórficamente Si en la MCM-41 y la SBA-15, respectivamente.

Palabras Claves: Nopol, Sn-SBA-15, Sn-MCM-41, $\beta$-pineno

\begin{abstract}
The catalytic activity of Sn-MCM-41 and Sn-SBA-15 for nopol synthesis from $\beta$-pinene and paraformaldehyde was compared in this work. The materials were prepared by the hydrothermal and the incipient wetness impregnation methods using stannous chloride as $\mathrm{Sn}$ precursor. In contrast to hydrothermal methods, impregnation leads to more active and selective materials for nopol production from $\beta$-pinene. $\beta$-Pinene conversion over Sn-MCM-41 and Sn-SBA-15 was similar. In the materials obtained by hydrothermal procedures, $\mathrm{Sn}(\mathrm{IV})$ species were detected as bulk $\mathrm{SnO}_{2}$ in $\mathrm{MCM}-41$ and isomorphously substituting Si in SBA-15.
\end{abstract}

Keywords: Nopol, Sn-SBA-15, Sn-MCM-41, $\beta$-pinene 


\section{Introducción}

El nopol se reconoce como un intermediario en la síntesis de productos utilizados en saborizantes, pesticidas, fragancias, detergentes, ceras y en general productos para el hogar (Bauer et al., 2001). También sobresale su aplicación en formulaciones biodegradables de pinturas reactivas, evitando el uso de compuestos menos amigables ambientalmente, tales como: cloruro de metileno, fenol, metanol, tolueno, acetona y compuestos cáusticos o corrosivos (Stevens, 1997). El nopol se obtiene principalmente mediante la reacción de condensación de Prins entre el $\beta$-pineno y el polímero anhidro del formaldehído, conocido como paraformaldehído, (Figura 1). El $\beta$-pineno es uno de los principales componentes del aceite de trementina, que se extrae de árboles de pino, y junto con el $\alpha$-pineno y el limoneno, es uno de los monoterpenos de mayor interés comercial por la posibilidad de ser transformado en compuestos de mayor valor agregado.

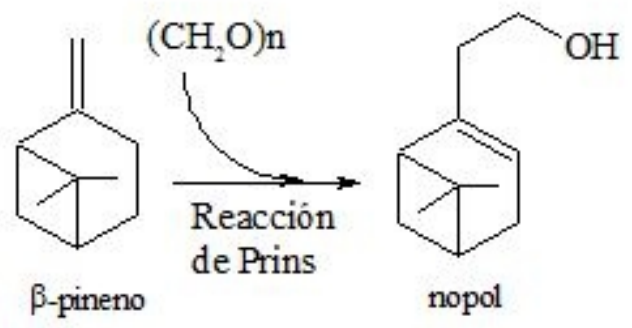

Figura 1. Síntesis de nopol mediante la reacción Prins

Los procesos tradicionales de síntesis de nopol involucran temperaturas alrededor de $200{ }^{\circ} \mathrm{C}$ a presión autógena o usando catalizadores ácidos Lewis homogéneos como el $\mathrm{ZnCl}_{2}$ a $120^{\circ} \mathrm{C}$ (Bain, 1946). Sin embargo, mediante el uso de materiales mesoporosos, con acidez Lewis conferida por especies de estaño o zinc, es posible efectuar la reacción a $90{ }^{\circ} \mathrm{C}$ con rendimientos comparables a los obtenidos mediante síntesis térmica, y las ventajas ambientales asociadas a la facilidad de recuperación del catalizador. Los materiales SnMCM-41 y Sn-SBA-15, sintetizados con $\mathrm{SnCl}_{4}$ como sal precursora de $\mathrm{Sn}$, son promisorios en la reacción de condensación de Prins (Villa de P. et al., 2002, Villa de P. et al., 2005, Corma \& Renz, 2007, Ramaswamy et al., 2008, Selvaraj \& Choe, 2010). El precursor $\mathrm{SnCl}_{2} \cdot 2 \mathrm{H}_{2} \mathrm{O}$, que es más seguro y estable que $\mathrm{SnCl}_{4}$, también se ha utilizado exitosamente en la obtención de materiales soportados en MCM-41 que han mostrado propiedades similares a los obtenidos con $\mathrm{SnCl}_{4}$ (Alarcón et al., 2009a, Alarcón et al., 2010); sin embargo, no se ha reportado la síntesis de nopol con Sn-SBA-15 preparado con cloruro estañoso. El método de impregnación húmeda incipiente es más sencillo que el procedimiento hidrotérmico. El primer método se considera un proceso postsíntesis, en el que se parte de un sólido que sirve de soporte de las especies activas, las cuales se introducen mediante contacto del sólido con una solución de las sustancias precursoras. El método hidrotérmico es un proceso de síntesis directa donde usualmente se parte de soluciones acuosas de las sustancias orgánicas e inorgánicas que producen precipitados o geles según las condiciones del medio, sometiéndolas a tratamientos térmicos bajo presión autógena. Se ha encontrado que al aumentar la concentración de catalizador y la temperatura de reacción, aumenta la conversión del $\beta$-pineno y disminuye la selectividad a nopol; adicionalmente, la conversión de $\beta$-pineno se favorece en tolueno y acetato de etilo mientras que la selectividad se favorece en solventes de mayor polaridad como el acetonitrilo y 2-butanona (Alarcón et al., 2010, Selvaraj \& Choe, 2010, Selvaraj \& Sinha, 2010).

Los materiales silíceos mesoporosos como la MCM-41 y SBA-15 se están estudiando desde la década de 1990. El alta área superficial y el diámetro de poro mayor a $2 \mathrm{~nm}$ potencializa sus aplicaciones como soportes en el área de la catálisis, especialmente con sustratos voluminosos. Es necesario introducir heteroelementos o complejos basados en metales para mejorar las propiedades ácidas (Tuel, 1999), requeridas en muchas de las transformaciones orgánicas. Comparativamente, SBA-15 tiene una estructura con paredes más gruesas y mayor estabilidad que la MCM-41, con valores típicos en el rango de $3-6 \mathrm{~nm}$ y $1-1.5 \mathrm{~nm}$, respectivamente (Galarneau et al., 2007; Meynen et al., 2009). No obstante, entre las estrategias para mejorar la estabilidad de la MCM-41 se encuentra la 
incorporación de heteroátomos, que minimizan la inestabilidad asociada con los grupos silanol de las mesoestructuras (Nishiyama et al., 2005). En esta contribución se comparan las características y el desempeño en la producción de nopol de Sn-SBA15 y Sn-MCM-41 sintetizadas con cloruro estañoso como fuente de Sn utilizando el método hidrotérmico y la impregnación húmeda incipiente.

\section{Sección experimental}

El material MCM-41 se obtuvo siguiendo el método reportado por Grün et al. (1999). El material SBA-15 utilizado para impregnación, se sintetizó con el método reportado por Mesa et al. (2003). SnSBA15-I y SnMCM41-I se sintetizaron por impregnación húmeda incipiente con $\mathrm{SnCl}_{2} \cdot 2 \mathrm{H}_{2} \mathrm{O}$ (Alarcón et al., 2010). En atmósfera inerte, $3 \mathrm{~mL}$ de solución de $\mathrm{SnCl}_{2} \cdot 2 \mathrm{H}_{2} \mathrm{O}$ (grado reactivo, Alfa-Aesar) en acetato de etilo (28.7 $\mu \mathrm{mol} \mathrm{Sn} / \mathrm{mL})$ se añadió gota a gota al soporte $(2 \mathrm{~g})$ y se mezcló. El solvente se evaporó a temperatura ambiente por $24 \mathrm{~h}$, y el sólido se calcinó a $550{ }^{\circ} \mathrm{C}$ durante $5 \mathrm{~h}$.

La síntesis por el método hidrotérmico de SnMCM41-H y SnSBA15-H se realizó siguiendo los procedimientos reportados previamente por el Grupo Catálisis Ambiental (Alarcón et al., 2009a) y por Ramaswamy et al. (2008), respectivamente. En una síntesis típica de Sn-MCM-41 se disolvieron $7.4 \mathrm{~g}$ de bromuro de miristil trimetil amonio (MTABr, 99 \% p, Aldrich) en $326 \mathrm{~mL}$ de agua desionizada y posteriormente $20 \mathrm{~mL}$ de $\mathrm{NH}_{4} \mathrm{OH}(28-30 \%$ p, EM Science).

El $\mathrm{SnCl}_{2} \cdot 2 \mathrm{H}_{2} \mathrm{O}(900 \mu \mathrm{mol})$ se adicionó como sólido. El pH se ajustó en $\sim 11.6$ con hidróxido de amonio y se adicionó gota a gota $(\sim 0.4 \mathrm{~mL} / \mathrm{min}) 28$ $\mathrm{g}$ de tetraetil ortosilicato ( $98 \% \mathrm{p}$, Acros Organics). La composición de 1 gel fue $\mathrm{SiO}_{2}: 0.007 \mathrm{SnO}_{2}: 0.152 \mathrm{MTABr}$ :

$2.8 \mathrm{NH}_{4} \mathrm{OH}: 141.2 \mathrm{H}_{2} \mathrm{O}$, la mezcla se agitó $1 \mathrm{~h}$ a temperatura ambiente. El sólido obtenido se lavó con suficiente agua desionizada, se secó a $100^{\circ} \mathrm{C}$ y se calcinó a $550{ }^{\circ} \mathrm{C}$ durante $5 \mathrm{~h}$. En una síntesis hidrotérmica típica de materiales Sn-SBA-15, $4 \mathrm{~g}$ de poli(etilen glicol)-poli(propilen glicol)poli(etilen glicol) (P123, $5800 \mathrm{~g} / \mathrm{mol}$, Aldrich) se añadieron a $30 \mathrm{~mL}$ de agua desionizada. Después de agitar durante $4 \mathrm{~h}$, se obtuvo una solución clara. Posteriormente, se adicionaron $70 \mathrm{~mL}$ de $\mathrm{HCl} 0.07$ M y la solución se agitó durante $2 \mathrm{~h}$. Luego se añadieron $9 \mathrm{~g}$ de tetraetilortosilicato $(98 \% \mathrm{p}$, Aldrich) y $0.2420 \mathrm{~g} \mathrm{de} \mathrm{SnCl}_{2} \cdot 2 \mathrm{H}_{2} \mathrm{O}$ (99\%, Aldrich) $\left(n_{S i} / n_{S n}=40\right)$. La mezcla resultante se agitó $24 \mathrm{~h} \mathrm{a}$ $40{ }^{\circ} \mathrm{C}$ y se dejó en un autoclave a $100{ }^{\circ} \mathrm{C}$ durante 24 h. El sólido obtenido se filtró, se lavó con agua desionizada y se secó a temperatura ambiente. Posteriormente, el material se calcinó en aire a $550^{\circ} \mathrm{C} \mathrm{K}$ durante $24 \mathrm{~h}$ para descomponer el copolímero tribloque.

El contenido de estaño se determinó por absorción atómica en un espectrómetro Thermo Elemental SOLAAR S4. El área superficial BET se determinó con medición de un solo punto en un equipo Micromeritics Autochem 2920; antes de la adsorción de nitrógeno $\left(5 \% \mathrm{~N}_{2} / \mathrm{He}\right)$ la muestra se trató a $150^{\circ} \mathrm{C}$ durante $30 \mathrm{~min}$.

El área superficial de algunas de las muestras también se determinó con el método BET a través de la isoterma de adsorción de nitrógeno a $77 \mathrm{~K}$ con un equipo Micromeritics ASAP2010; las muestras fueron pre-tratadas a $250^{\circ} \mathrm{C}$. El diámetro de poro promedio se estimó según la distribución de tamaño de poro por el método BJH.

Los análisis de difracción de rayos $X$ (DRX) de las muestras basadas en MCM-41 se realizaron en un difractómetro AXS Brucker provisto con una lámpara de cobre a $2 \% \mathrm{~min}$. El análisis SEM se realizó en un equipo JEOL modelo JSM-6490LV.

La reducción con temperatura programada (TPR) se realizó en el equipo Autochem 2920, activando $0.05 \mathrm{~g}$ de muestra en $\mathrm{He}(50 \mathrm{~mL} / \mathrm{min})$ hasta $550{ }^{\circ} \mathrm{C}$ $\left(10^{\circ} \mathrm{C} / \mathrm{min}\right)$ durante $30 \mathrm{~min}$, la muestra se enfrió a $40^{\circ} \mathrm{C}$ y posteriormente se trató en presencia de una mezcla $5 \% \mathrm{H}_{2} / \operatorname{Ar}(25 \mathrm{~mL} / \mathrm{min})$ a $1000{ }^{\circ} \mathrm{C}$ con una velocidad de calentamiento de $10{ }^{\circ} \mathrm{C} / \mathrm{min}$. Para corregir las señales se realizaron dos blancos, el primero empleando Ar puro en vez de la mezcla $\mathrm{H}_{2} / \mathrm{Ar}$ y el segundo sin muestra en el reactor de cuarzo. 
El consumo de hidrógeno se estimó mediante calibración con varios pesos conocidos de muestras de $\mathrm{CuO}$ y bajo las condiciones típicas del análisis. Los análisis de TPD se realizaron con 50 $\mathrm{mg}$ de $\mathrm{Sn}-\mathrm{MCM}-41$, realizando la activación en $\mathrm{He}(50 \mathrm{~mL} / \mathrm{min})$ hasta $550{ }^{\circ} \mathrm{C}\left(10^{\circ} \mathrm{C} / \mathrm{min}\right)$ durante $30 \mathrm{~min}$. Posteriormente, las muestras se enfriaron por convección a $40{ }^{\circ} \mathrm{C}$ y se saturaron con amoníaco $(0.3 \%$ en $\mathrm{He}, 50 \mathrm{~mL} / \mathrm{min})$ durante 90 min; el amoníaco fisiadsorbido se desorbió con $\mathrm{He}$ $(30 \mathrm{~mL} / \mathrm{min})$ a $40^{\circ} \mathrm{C}$ durante $1 \mathrm{~h}$, posteriormente se calentó la muestra a $10^{\circ} \mathrm{C} / \mathrm{min}$ hasta $800^{\circ} \mathrm{C}$.

En una reacción típica se adicionó el catalizador (6 ó $12 \mathrm{mg}$ ), el paraformaldehído $(29.2 \mathrm{mg})$ y $1 \mathrm{~mL}$ de solución $0.46 \mathrm{M}$ de $\beta$-pineno en tolueno a un recipiente de vidrio de $2 \mathrm{~mL}$ que se sumergió en un baño de aceite cuya temperatura se controló con un equipo marca ETS-D4 Fuzzy. La mezcla de reacción se homogenizó magnéticamente, analizándose muestras de reacción entre 5 min y $24 \mathrm{~h}$. Las sustancias de interés se cuantificaron por cromatografía de gases con dodecano como estándar interno (Alarcón et al., 2010). La conversión y selectividad se calcularon con la Ec. (1) y Ec. (2), respectivamente

$$
\begin{aligned}
\% X=\frac{\left(C_{i}-C_{f}\right)_{\beta-\text { pineno }}}{\left(C_{i}\right)_{\beta-\text { pineno }}} 100 \% \\
\% S=\frac{\left(C_{f}\right)_{\text {nopol }}}{\left(C_{i}-C_{f}\right)_{\beta-\text { pineno }}} 100 \%
\end{aligned}
$$

Donde $X$ es conversión, $S$ selectividad y $C_{i}$ y $C_{f}$ son las concentraciones inicial y final, respectivamente.

El TON (del término en inglés Turn Over Number) y el TOF (del término en inglés Turn Over Frequency) se calcularon con las ecuaciones (3) y (4), respectivamente.

$$
\begin{gathered}
\text { TON }=\frac{\left(C_{i}\right)_{\beta-\text { pineno }} * \% X}{100 * n_{S n}} \\
\text { TOF }=\frac{\text { TON }}{t}
\end{gathered}
$$

Donde $n_{S n}$ son las moles de Sn en la cantidad de catalizador utilizado para la reacción, $t$ es el tiempo en el que se alcanza la conversión $\% X$.

Para evaluar la lixiviación del estaño de SnSBA15-H la reacción se llevó a cabo a una conversión menor al 10\%, luego el catalizador se separó del líquido a la temperatura de reacción utilizando un filtro Acrodisc ${ }^{\circledR}$ PSF. Los análisis de lixiviación de los materiales basados en MCM-41 fueron previamente reportados (Alarcón et al., 2010), comprobándose que a las condiciones de reacción el Sn no se lixivia de Sn-MCM-41 obtenido por métodos hidrotérmicos y por impregnación.

\section{Resultados y discusión}

El área superficial BET de los materiales sintetizados (Tabla 1) es típica de materiales mesoporosos obtenidos con procesos de síntesis similares 500-800 y $900-1550 \mathrm{~m}^{2} / \mathrm{g}$ para SBA-15 y MCM-41, respectivamente (Meynen et al., 2009).

Las isotermas de adsorción de $\mathrm{N}_{2}$ de SnSBA15-H y los materiales basados en MCM-41 se presentan en la Figura 2. SnSBA15-H exhibe una isoterma tipo IV según la clasificación de la IUPAC con un lazo de histéresis característico de los materiales mesoporosos (Neimark et al., 2008).

Las isotermas de MCM-41 y SnMCM41-I también se pueden clasificar como tipo IV, sin lazos de histéresis, con un aumento pronunciado en la cantidad adsorbida a una presión relativa alrededor de 0.2 , las cuales son características de materiales porosos cuyo diámetro de poro se encuentra en la frontera de los microporos y los mesoporosos (Miyata et al., 2003). Jaronieck et al. (2001) ha relacionado el desplazamiento del lazo de condensación capilar a menores presiones relativas con la disminución del diámetro de poro.

El diámetro de poro promedio estimado por el método BJH (Tabla 1) se encuentra en el rango de los mesoporosos para los materiales basados en MCM-41 (alrededor de $2 \mathrm{~nm}$ ) y los materiales basados en SBA-15 ( 6.3 y $6.7 \mathrm{~nm}$ ). Los valores 
obtenidos para SnSBA15-H y materiales basados en MCM-41 son similares a los valores calculados con la aproximación $d_{p}=4 V_{p} / A_{p}$ por el método BET.
Los análisis DRX de los materiales basados en MCM-41 (Figura 3), exhiben la señal amplia característica de la mesoestructura de la MCM-41 centrada en alrededor de $2 \theta=2.6^{\circ}$ (Grün et al.,

Tabla 1. Efecto del método de sintesis de Sn-SBA-15 y Sn-MCM-41 en la producción de nopol*

\begin{tabular}{|c|c|c|c|c|c|c|c|c|c|c|}
\hline Catalizador & $\begin{array}{l}\text { Área BET }{ }^{a} \\
\left(\mathrm{~m}^{2} / \mathrm{g}\right)\end{array}$ & $\mathrm{dp}(\mathrm{nm})$ & $\begin{array}{l}\mathrm{Si} / \mathrm{Sn} \\
\text { nominal }\end{array}$ & $\begin{array}{l}\mathrm{Si} / \mathrm{Sn} \\
\text { medido }\end{array}$ & $\begin{array}{l}\text { Contenido } \\
\text { de Sn, \% } \\
\text { p/p }\end{array}$ & $\begin{array}{l}\% \text { de Sn } \\
\text { incorporado }^{c}\end{array}$ & $\% X^{d}$ & $\% \mathrm{~S}^{\mathrm{d}}$ & $\begin{array}{l}\text { TOF }\left(h^{-1}\right) \\
\text { e }\end{array}$ & $\mathrm{TON}^{\mathrm{d}}$ \\
\hline SBA-15 & 944 & 6.3 & & $\infty$ & 0 & 0 & 6.9 & 42 & -- & -- \\
\hline SnSBA15-H & $697(589)$ & 6.7 & 40 & 64 & 3.09 & 62.5 & 55 & 77 & $163 \pm 9$ & $81 \pm 4$ \\
\hline SnSBA15-I & nd (524) & nd & 387 & 800 & 0.25 & 48.4 & 47 & 83 & $1721 \pm 102$ & $860 \pm 51$ \\
\hline MCM-41 & $924(929)$ & 2.0 & $\infty$ & $\infty$ & 0 & 0 & 4.6 & 37 & -- & -- \\
\hline SnMCM41-I & 934 (1177) & 2.1 & 387 & 694 & 0.28 & 55.8 & 51 & 83 & $1667 \pm 95$ & $834 \pm 47$ \\
\hline SnMCM41-H & $1323(1315)$ & 1.9 & 149 & 387 & 0.43 & 38.5 & 53 & 78 & $1128 \pm 63$ & $564 \pm 31$ \\
\hline
\end{tabular}

nd: no analizado, dp: diámetro de poro* Condiciones de reacción: $12 \mathrm{mg}$ de catalizador, $1 \mathrm{~mL}$ de solución $0.46 \mathrm{M}$ de $\beta$-pineno en tolueno, relación paraformaldehído: $\beta$-pineno $=2: 1,90{ }^{\circ} \mathrm{C}, 30 \mathrm{~min} .{ }^{\mathrm{a}} \mathrm{El}$ valor en paréntesis corresponde al área determinada con un solo punto. ${ }^{\mathrm{b}}$ Determinado por absorción atómica, AA. ${ }^{\mathrm{c}} \%$ de $\mathrm{Sn}$ incorporado $=(\mathrm{Si} / \mathrm{Sn}$ nominal $) /(\mathrm{Si} / \mathrm{Sn}$ medido $) * 100 \%$. ${ }^{\mathrm{d}}$ La desviación estándar de las medidas de conversión y selectividad fue alrededor de 2 y 4 unidades, respectivamente. ${ }^{~ L a ~ d e s v i a c i o ́ n ~ e s t a ́ n d a r ~ d e ~ T O N ~ y ~ T O F ~ s e ~ e s t i m o ́ ~ c o n ~ l a ~ p r o p a g a c i o ́ n ~ d e ~ e r r o r e s, ~}$ empleando las desviaciones experimentales de la conversión, concentración inicial de $\beta$-pineno y carga de metal; se tomó el error relativo de contenido de Sn igual a $4 \%$.

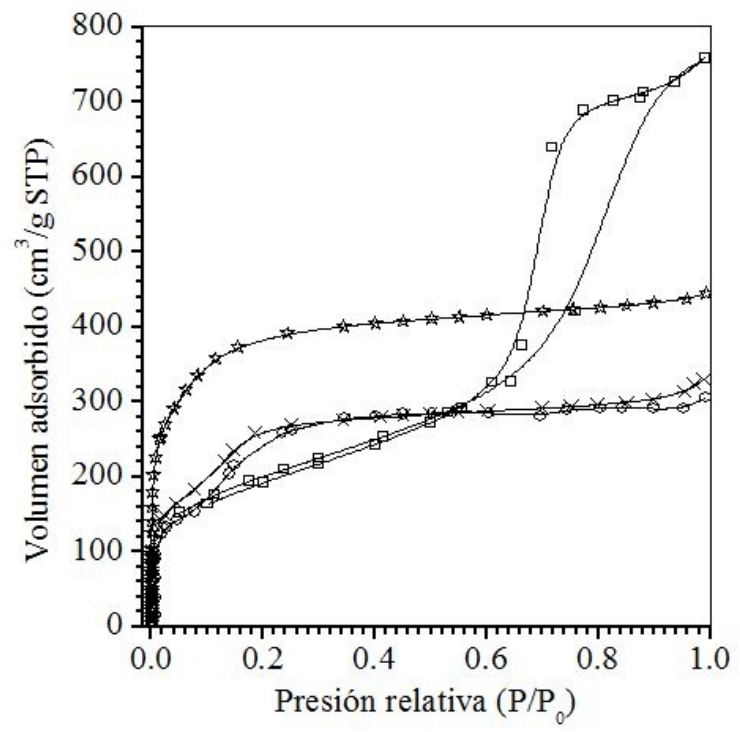

Figura 2. Isotermas de fisisorción de nitrógeno de SnSBA15-Hy materiales basados en MCM-41.

$$
\begin{aligned}
& -\square-\text { SnSBA15-H } \quad \text { - - MCM-41 } \longrightarrow \text { SnMCM41-I } \\
& \text { 一-SnMCM41-H }
\end{aligned}
$$

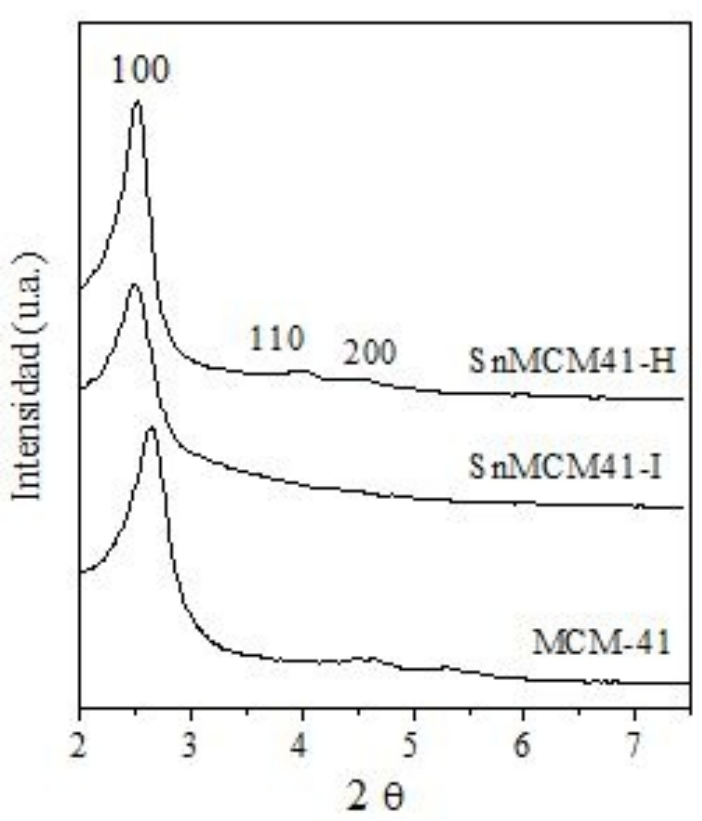

Figura 3. Difracción de rayos X de los materials basados en $M C M-41$ 
1999). El arreglo hexagonal de los canales mesoporosos de la MCM-41 se evidencia por la presencia de los planos de reflexión $d_{100}, d_{110} \mathrm{y} d_{200}$. Sin embargo, después del proceso post-síntesis no se observan los picos correspondientes a $d_{110}$ y $d_{200}$ en el material SnMCM41-I. La morfología de los materiales por SEM (Figura 4), exhiben la naturaleza amorfa de los materiales basados en MCM-41. En los materiales basados en SBA-15 se aprecian las formas de esferas, de tamaño no uniforme, y rollos en los materiales por impregnación y por síntesis directa, respectivamente (Meynen et al., 2009). La impregnación de estaño y posterior tratamiento térmico alteró la morfología original de esferas uniformes de la SBA-15 (Figura 4c).

Aunque para la síntesis de los materiales por el
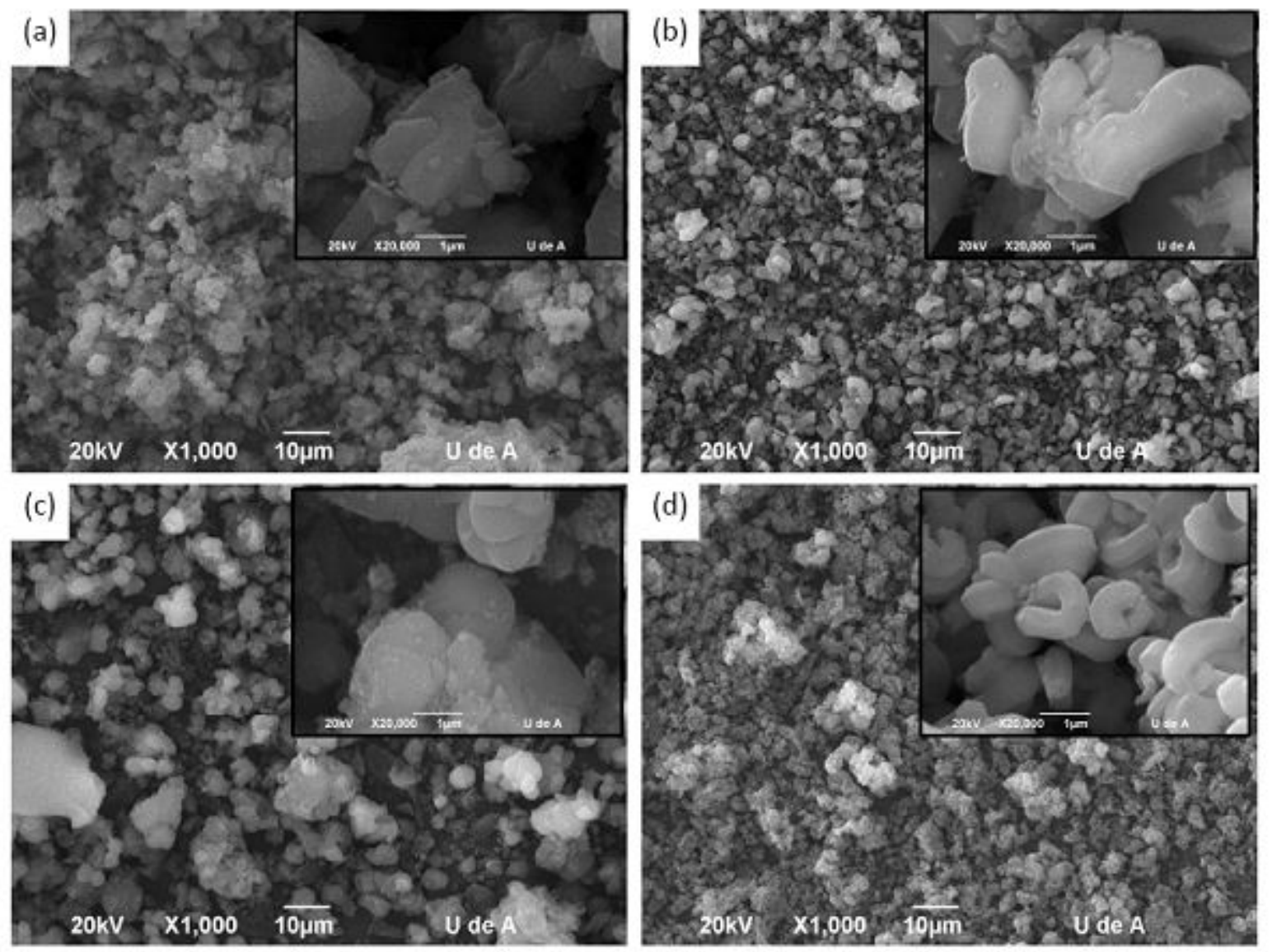

Figura 4. Microscopía electrónica de barrido, SEM, de los materiales de interés. a) SnMCM41-I, b) SnMCM41-H, c) SnSBA15-Iyd) SnSBA15-H. 
Coherente con trabajos anteriores (Alarcón et al., 2009a, Alarcón et al., 2009b), el análisis de TPR de las especies de estaño soportados tanto en SBA-15 como MCM-41 sugiere la presencia de especies reducibles en los materiales obtenidos por síntesis hidrotérmica (Figura 5), mientras que los materiales sintetizados por impregnación no exhiben señales características de óxidos de estaño; la baja concentración de $\mathrm{Sn}$ en los materiales preparados por el método de impregnación podría ser también causa de la ausencia de picos de reducción de las especies de $\mathrm{Sn}$. El pico de reducción a $657^{\circ} \mathrm{C}$ en el material de Sn-MCM-41 se ha atribuido a agregados de óxidos de estaño, presumiblemente en las paredes de la MCM-41 (Alarcón et al., 2009a); lo cual también se ha confirmado por análisis UV-vis (Alarcón et al., 2010). Burch et al. (2000) asociaron los picos de reducción a temperaturas superiores a $600{ }^{\circ} \mathrm{C}$ a agregados de óxidos de estaño (del término en inglés "bulk $\mathrm{SnOx}$ species"). El TPR de $\mathrm{SnO}_{2}$ puro usualmente muestra una única señal alrededor de $780{ }^{\circ} \mathrm{C}$ (Rumyantseva et al., 2006; Park et al., 1999).

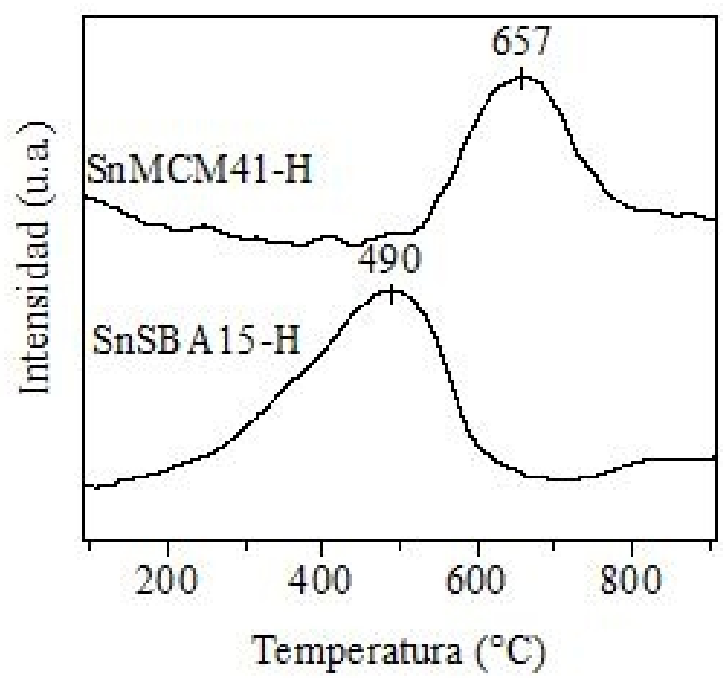

Figura 5. Reducción con temperatura programada $\mathrm{de}_{2}$ de los materiales sintetizados hidrotérmicamente

A temperaturas por debajo de $600{ }^{\circ} \mathrm{C}$ Burch et al. (2000) postuló la presencia de películas delgadas o agregados de tamaño pequeño de óxidos de estaño. A este tipo de especies se les atribuye la actividad en la producción de nopol en los materiales de Sn-MCM-41 post-síntesis (Alarcón et al., 2010). El TPR de SnSBA15-H exhibe solo una señal principal centrada en $490{ }^{\circ} \mathrm{C}$, que presumiblemente se puede atribuir a la reducción parcial de Sn(IV) tetraedral isomorfícamente sustituido a Sn(II) (Chaudhari et al., 1999), como fue reportado por Nava \& Viveros (1999) que asignaron las señales TPR a 600 y $700{ }^{\circ} \mathrm{C}$, en materiales de estaño soportado en silica, a la reducción total probablemente en dos etapas de $\mathrm{SnO}_{2}$ a estaño metálico.

En SnSBA15-H, probablemente la mayor parte del Sn se encuentre en sitios de la SBA-15 en el que su estado de reducción más estable sea $\mathrm{Sn}$ (II), como en sitios de sustitución isomórfica de Si. El medio ácido de la síntesis de SnSBA15-H favorece mayoritariamente la incorporación de $\mathrm{Sn}(\mathrm{IV})$ en la estructura del catalizador, mientras el medio básico de la síntesis de SnMCM41-H promueve la formación de agregados de óxidos de estaño y una baja proporción de especies de $\mathrm{Sn}(\mathrm{OSi})_{4}$ a las que se le atribuye la actividad catalítica (Alarcón et al., 2010).

El contenido de especies reducibles en SnMCM41-H y SnSBA15-H se estimó considerando el consumo de hidrógeno y las asignaciones de los picos de TPR. Así, se requieren 2 moles de hidrógeno para reducir totalmente el $\mathrm{SnO}_{2}$ en la señal de $657^{\circ} \mathrm{C}$ y $1 \mathrm{~mol}$ de hidrógeno para la reducción parcial de $\mathrm{Sn}(\mathrm{IV})$ a $\mathrm{Sn}$ (II) en la señal a $490{ }^{\circ} \mathrm{C}$, obteniéndose contenidos de estaño de 0.58 y $2.45 \%$ p/p para SnMCM41-H y SnSBA15-H, respectivamente. Aunque estos valores difieren de los obtenidos por AA (Tabla 1), pueden corresponder a un estimativo adecuado de la asignación de las especies de estaño.

En la Tabla 1 se compara la actividad de los materiales Sn-SBA-15 y Sn-MCM-41 sintetizados por el método hidrotérmico y de impregnación con el precursor cloruro estañoso. La conversión obtenida con los materiales preparados por el método hidrotérmico fue superior a la obtenida con los materiales preparados por impregnación, siendo mayor la diferencia al utilizar SBA-15 como soporte. La 
selectividad a nopol fue mayor con los materiales obtenidos por impregnación y no varió con el soporte.

El orden de conversión se relaciona con la cantidad de metal incorporado (Tabla 1), sin embargo, dicha relación no es proporcional. Se ha encontrado en materiales sintetizados por procesos post-síntesis que no existen diferencias significativas en la conversión con cargas de metal en el rango 16 - $500 \mu \mathrm{mol} \mathrm{Sn/g}$ catalizador $(0.18$ $5.9 \%$ p/p Sn) (Villa De P. et al., 2005, Alarcón et al., 2009b).

Los materiales del tipo SnMCM41-H no tienen un patrón tan definido, con tendencia a disminuir la conversión con la menor carga de metal y un óptimo de conversión y selectividad con relación $\mathrm{Si} / \mathrm{Sn}$ alrededor de 387; con cargas superiores de metal la conversión se puede afectar negativamente (Alarcón et al., 2009a). Ya que se obtuvo la misma conversión y selectividad con diferentes cantidades de Sn en los materiales hidrotérmicos, se podría sugerir que las especies de Sn en SnMCM41-H son más reactivas que las que se encuentran en SnSBA15-H, pues para la misma cantidad de catalizador, este presentaba mayor cantidad de $\mathrm{Sn}$, indicando que aunque para la síntesis de ambos materiales se utilizaron procedimientos hidrotérmicos, las variaciones en estos métodos pueden ocasionar la formación de diferentes especies de Sn.

El seguimiento de la conversión y la selectividad con los catalizadores SnSBA15-H y SnMCM41-I indica que para tiempos superiores a $3 \mathrm{~h}$, la conversión (Figura 7a) obtenida con SnMCM41-I es ligeramente superior a la alcanzada con SnSBA15-H, y que la selectividad (Figura $7 b$ ) con el material SnMCM41-I es superior a la de SnSBA15-H en todo el rango estudiado. En términos de actividad expresada como TOF (de las siglas en inglés Turnover Frequency) promedio o TON (de las siglas en inglés Turnover Number), los materiales obtenidos por impregnación con ambos soportes presentan mejor desempeño que los obtenidos mediante síntesis hidrotérmica. La prueba de lixiviación de SnSBA15-H indicó que después de separar el catalizador del medio de reacción no se presentó aumento ni en la conversión ni en la producción de nopol.
La variación de conversión de $\beta$-pineno con el tiempo reportada por Ramaswamy et al. (2008) para Sn-SBA-15 obtenido por síntesis hidrotérmica, es similar a la presentada en la Figura 7a. Selvaraj \& Sinha (2010) compararon la actividad de materiales Sn-SBA-15 sintetizados por los procesos post-síntesis CVD (de las siglas en inglés Chemical Vapor Deposition) e impregnación, con Sn-MCM-41 obtenida por síntesis hidrotérmica con diversas fuentes de estaño. Los autores encontraron que la selectividad era mayor con los materiales SnSBA-15 obtenidos por CVD de $\mathrm{SnCl}_{4}$ que por impregnación de disoluciones de $\mathrm{SnCl}_{4} \cdot 5 \mathrm{H}_{2} \mathrm{O}$ en etanol y de acetato de estaño en solución acidificada con $\mathrm{HCl}$, y mayor a la observada con Sn-MCM-41 producida por síntesis hidrotérmica con $\mathrm{SnCl}_{4} \cdot 5 \mathrm{H}_{2} \mathrm{O}$. El TOF promedio a $6 \mathrm{~h}$ de reacción con dichos materiales fue del orden de $440 \mathrm{~h}^{-1}$, comparable al valor obtenido $\left(370 \pm 19 \mathrm{~h}^{-1}\right)$ para SnMCM41-I (Figura 7a).

Selvaraj \& Choe (2010), reportaron que con materiales Sn-MCM-41 obtenidos por síntesis hidrotérmica con $\mathrm{SnCl}_{4} \cdot 5 \mathrm{H}_{2} \mathrm{O}$ y una relación $\mathrm{Si} / \mathrm{Sn}$ nominal de 40 se obtuvo una conversión de $91 \%$ y selectividad de $82 \%$, respectivamente, bajo condiciones de reacción similares a las reportadas en esta contribución (Tabla 1), pero a $6 \mathrm{~h}$ de reacción; esto equivale a un TOF de $289 \mathrm{~h}^{-1}$. En el mismo reporte con Sn-SBA-15 hidrotérmico ( $\mathrm{Si} / \mathrm{Sn}$ real de 53.6) se obtuvo un TOF de $14 \mathrm{~h}^{-1}$ a conversión y selectividad de 63 y $77 \%$, respectivamente; el cuál es menor al obtenido (33 $\pm 2 \mathrm{~h}^{-1}$ ) bajo las condiciones de la Figura 7 .

Las diferencias de la producción de nopol y la tendencia a la formación de subproductos de catalizadores Sn-SBA-15 han sido atribuidas al ambiente de las distintas especies de estaño y a sus propiedades ácidas (Selvaraj \& Choe, 2010; Selvaraj \& Sinha, 2010). Los estudios de acidez realizados en Sn-MCM-41 (Alarcón et al., 2009a; Alarcón et al., 2009b), indican que los sitios Lewis de acidez débil y media, preferiblemente, son responsables de la producción de nopol.

Estas características también fueron analizadas por Ramaswamy et al. (2008) en materiales SnSBA-15 obtenidos por impregnación y síntesis 
hidrotérmica, encontrando diferencias en los análisis TPD de los materiales sintetizados por diferentes métodos y con diferentes carga de metal. SBA-15 impregnada con $\mathrm{SnCl}_{4}$ mostró señales amplias correspondientes a sitios de acidez débil y media en proporciones similares. Sn-SBA-15 obtenido hidrotérmicamente bajo condiciones ácidas presentó señales en las mismas posiciones pero con mayor proporción de sitios débiles, proponiendo que no solo la acidez del material sino también la dispersión y estado de coordinación favorecen la reacción de producción de nopol.

El TPD de SnMCM41-I y SnMCM41-H (Figura 6) muestra un rasgo más similar al de los materiales impregnados reportados por Ramaswamy et al. (2008), no obstante con mayor proporción de sitios de acidez media. Considerando solo las dos señales de acidez débil y media por encima de $180{ }^{\circ} \mathrm{C}$ (Alarcón et al., 2009a) visibles en la Figura 6, la acidez total para el soporte MCM-41, SnMCM41$\mathrm{H}$ y SnMCM41-I se encuentra alrededor de 0.020 ,

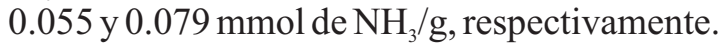
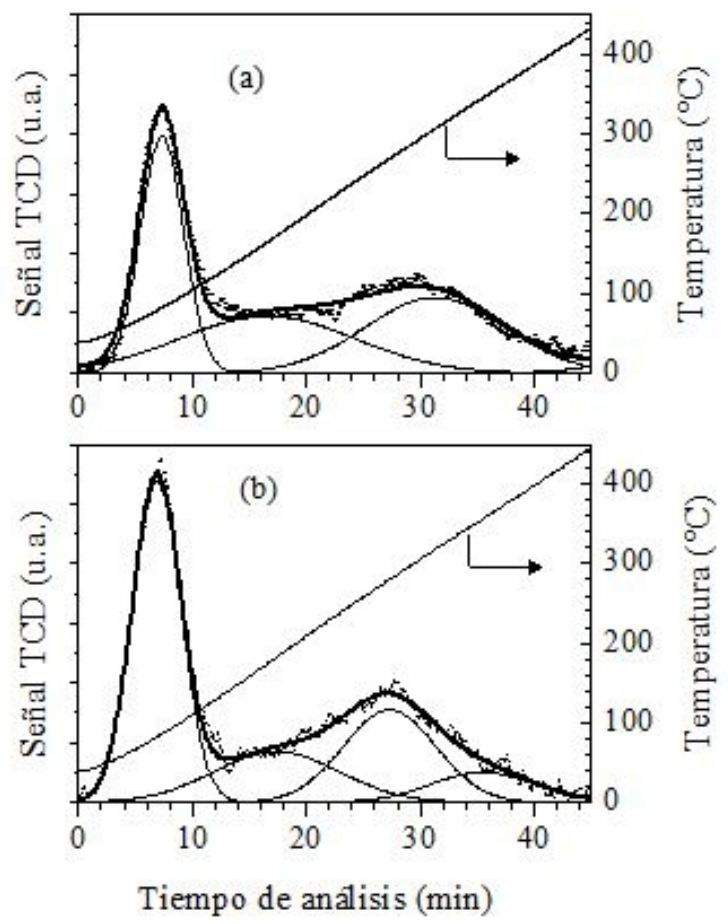

Figura 6. Análisis de TPD y deconvolución de las curvas de SnMCM41-I (a) y SnMCM41-H(b).
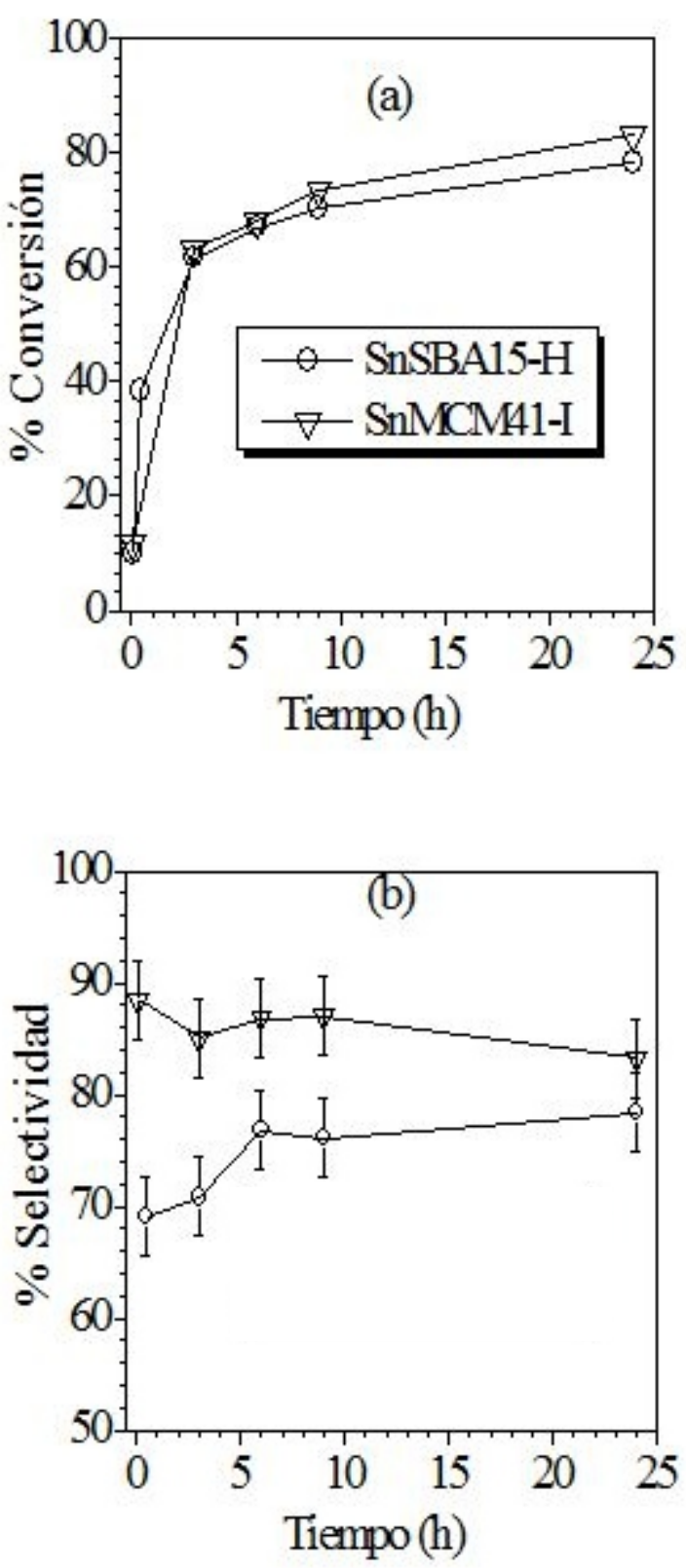

Figura 7. Conversión (a) y selectividad (b) vs tiempo. Condiciones de reacción: catalizador $6 \mathrm{mg}, 1 \mathrm{~mL}$ de solución de $\beta$-pineno en tolueno 0,46 $M$, relación paraformaldehído: $\beta$-pineno $=2: 1,90{ }^{\circ} \mathrm{C}$. El tamaño de los símbolos para la conversión es del mismo orden de magnitud que la respectiva desviación estándar. $\rightarrow$ SnSBA15-H, $\rightarrow$ - SnMCM41-I.

Estos valores son inferiores a los reportados por Ramaswamy et al. (2008) para los materiales obtenidos por síntesis hidrotérmica que se encuentran entre 0.094 y 0.229 , respectivamente. 


\section{Conclusiones}

El cloruro estañoso $\mathrm{SnCl}_{2} \cdot 2 \mathrm{H}_{2} \mathrm{O}$ se puede utilizar como fuente de $\mathrm{Sn}$ en la síntesis de Sn-SBA-15 y Sn-MCM-41, tanto por síntesis hidrotérmica como por impregnación incipiente. Mediante síntesis hidrotérmica, en soportes de MCM-41 y SBA-15, se producen especies de Sn(IV) formando, principal y respectivamente, agregados de $\mathrm{SnO}_{2}$ y substituyendo isomórficamente el $\mathrm{Si}$ en la estructura.

El método de impregnación es más adecuado para la síntesis de nopol, ya que la selectividad a este compuesto es mayor que con los materiales preparados por el método hidrotérmico. En términos del TOF los materiales impregnados son más activos respecto a los obtenidos por método hidrotérmico. No se encontraron diferencias significativas en la conversión y selectividad alcanzadas con los dos materiales sintetizados por impregnación.

\section{Agradecimientos}

Los autores agradecen el apoyo financiero de Colciencias y la Universidad de Antioquia a través del Centro de Excelencia CENIVAM, Contrato RC No 245-2011, y a la UdeA mediante la estrategia de sostenibilidad 2009 - 2011. E.A. también agradece a Colciencias la beca doctoral.

\section{Referencias}

Alarcón, E., Villa, A. L. \& Montes de C., C. (2009a). Efecto de las condiciones de síntesis hidrotérmica de Sn-MCM-41 en la producción de nopol. Revista Facultad de Ingeniería, Universidad de Antioquia (49), 19-29.

Alarcón, E. A., Correa, L., Montes de C., C. \& Villa, A. L. (2010). Nopol production over SnMCM-41 synthesized by different procedures Solvent effects. Microporous and Mesoporous Materials, 136, 59-67.

Alarcón, E. A., Villa, A. L. \& Montes de C., C. (2009b). Characterization of Sn- and Zn-loaded MCM-41 catalysts for nopol synthesis.
Microporous and Mesoporous Materials, 122, 208-215.

Bain, J. P. (1946). Nopol. I. The reaction of $\beta$ pinene with formaldehyde. Journal of the American Chemical Society, 68, 638-641.

Bauer, K., Garbe, D. \& Suburg, H. (2001). Common Fragrance and Flavor Materials. Preparation, Properties and Uses, Weinheim, VCH Verlagsgesellschaft.

Burch, R., Caps, V., Gleeson, D., Nishiyama, S. \& Tsang, S. C. (2000). Nanoscopic tin-oxygen linings on mesoporous silica as a novel catalyst for organic hydrogen transfer reaction. Applied Catalysis A: General, 194-195, 297-307.

Chaudhari, K., Das, T. K., Rajmohanan, P. R., Lazar, K., Sivasanker, S. \& Chandwadkar, A. J. (1999). Synthesis, Characterization, and Catalytic Properties of Mesoporous Tin-Containing Analogs of MCM-41. Journal of Catalysis, 183, 281-291.

Corma, A. \& Renz, M. (2007). Water-resistant Lewis-acid sites: Carbonyl-ene reactions catalyzed by tin-containing, hydrophobic molecular sieves. Arkivoc, 2007, 40-48.

Galarneau, A., Nader, M., Guenneau, F., Di Renzo, F. \& Gedeon, A. (2007). Understanding the Stability in Water of Mesoporous SBA-15 and MCM-41. The Journal of Physical Chemistry $C$, $111,8268-8277$.

Gonzebach, C., Jordan, M., \& Yunick, R. (1964). Terpene Resins. In: H.F. Mark, N.G. Gaylord, N.M. Bikales (editors), Encyclopedia of polymer science and technology plastics resins rubbers fibers, John Wiley \& sons, (Volume 13).

Grün, M., Unger, K. K., Matsumoto, A. \& Tsutsumi, K. (1999). Novel pathways for the preparation of mesoporous MCM-41 materials: control of porosity and morphology. Microporous and Mesoporous Materials, 27, 207-216.

Jadhav, S.V., Jinka , K.M. \& Bajaj , H.C. (2012). Nanosized sulfated zinc ferrite as catalyst for the 
synthesis of nopol and other fine chemicals. Catalysis Today, artículo en prensa doi:10.1016/j.cattod.2012.01.028.

Jaroniec, M., Kruk, M., Shin, H.J., Ryoo, R., Sakamoto, Y., \& Terasaki, O. (2001). Comprehensive characterization of highly ordered MCM-41 silicas using nitrogen adsorption, thermogravimetry, X-ray diffraction and transmission electron microscopy. Microporous and Mesoporous Materials, 48 (1-3), 127-134.

Mesa, M., Sierra, L., López, B., Ramirez, A. \& Guth, J.-L. (2003). Preparation of micron-sized spherical particles of mesoporous silica from a triblock copolymer surfactant, usable as a stationary phase for liquid chromatography. Solid State Sciences, 5, 1303-1308.

Meynen, V., Cool, P. \& Vansant, E. F. (2009). Verified syntheses of mesoporous materials. Microporous and Mesoporous Materials, 125, 170-223.

Miyata, T., Endo, A., Ohmori, T., Akiya, T. \& Nakaiwa, M. (2003). Evaluation of pore size distribution in boundary region of micropore and mesopore using gas adsorption method. Journal of Colloid and Interface Science 262, 116-125.

Nava, N. \& Viveros, T. (1999). Structural studies of supported tin catalysts. Hyperfine Interactions, $122,147-153$.

Nava, N. \& Viveros, T. (2000). Effect of the support on the structural characteristics of tin. Journal of Radioanalytical and Nuclear Chemistry, 243 (3), 689-696.

Neimark, A.V., Sing, K.S.W. \& Thommes, M. (2008). Characterization of Solid Catalysts: Surface Area and Porosity. In: G. Ertl, H. Knözinger, J. Weitkamp (editors), Handbook of Heterogeneous Catalysis, Wiley-VCH, Weinheim, (Chapter 3).

Nishiyama, N., Yamaguchi, M., Nishiyama, Y., Egashira, Y. \& Ueyama, K. (2005). Enhancement of structural stability of mesoporous silica via infiltration of $\mathrm{SnCl}_{4}$ vapor. Journal of NonCrystalline Solids, 351, 3218-3224.
Park, P.W., Kung, H.H., Kim, D.W., Kung, M.C. (1999). Characterization of $\mathrm{SnO}_{2} / \mathrm{Al}_{2} \mathrm{O}_{3}$ lean NOx catalysts. Journal of Catalysis, 184 (2), 440-454.

Ramaswamy, V., Shah, P., Lazar, K. \& Ramaswamy, A. V. (2008). Synthesis, characterization and catalytic activity of Sn-SBA15 mesoporous molecular sieves. Catalysis Surveys from Asia, 12, 283-309.

Rumyantseva, M., Kovalenko, V., Gaskov, A., Makshina, E., Yuschenko, V., Ivanova, I., Ponzoni, A., Faglia, G., Comini, E. (2006). Nanocomposites $\mathrm{SnO}_{2} / \mathrm{Fe}_{2} \mathrm{O}_{3}$ : Sensor and catalytic properties. Sensors and Actuators B, 118 (1-2), 208-214.

Selvaraj, M. \& Choe, Y. (2010). Well ordered twodimensional SnSBA-15 catalysts synthesized with high levels of tetrahedral tin for highly efficient and clean synthesis of nopol. Applied Catalysis A: General, 373, 186-191.

Selvaraj, M. \& Sinha, P. K. (2010). Highly selective and clean synthesis of nopol over wellordered mesoporous tin silicate catalysts. New Journal of Chemistry, 34, 1921-1929.

Stevens, E. (1997). Paint stripping compositions and methods. United States patent application No. $5,597,788$.

Swift , K.A.D. (2004). Catalytic Transformations of the Major Terpene Feedstocks. Topics in Catalysis, 27 143-155.

Tuel, A. (1999). Modification of mesoporous silicas by incorporation of heteroelements in the framework. Microporous and. Mesoporous Materials 27, 151-169.

Villa de P., A. L., Alarcón, E. \& Montes de C., C. (2005). Nopol synthesis over Sn-MCM-41 and Sn-kenyaite catalysts. Catalysis Today, 107-108, 942-948.

Villa de P., A. L., Alarcón, E. \& Montes de C., C. (2002). Synthesis of nopol over MCM-41 catalysts. Chemical Communications, 2654-2655. 
Xiao, Z., Li, Z., Chen, J., Fu, Y., \& Wang, M. (1999). Analysis and identification of the structure of the main by-product in the synthetic reaction of nopol. Journal of Jiangxi Normal University 23 (4), 360-362. 